\title{
Investigation of the direct/indirect exciton transition in the double quantum well system based on $\mathrm{Cd}_{1-\mathrm{y}} \mathrm{Mg} \mathrm{Te} / \mathrm{Cd}_{1-\mathrm{x}} \mathrm{Mn}_{\mathrm{x}} \mathrm{Te}$ in applied magnetic field
}

\author{
S.B. Lev, V.I. Sugakov, and G.V. Vertsimakha \\ Institute for Nuclear Research, NAS of Ukraine \\ 47, prospect Nauky, Kyiv 03680, Ukraine \\ E-mail:sugakov@kinr.kiev.ua
}

\begin{abstract}
The energy levels, wave functions, and lifetimes of direct and indirect excitons in a CdTe-based double quantum well system with non-magnetic (Mg) and magnetic (Mn) ions in a magnetic field are found. It is shown that, in an external magnetic field greater than some critical value, the lowest excited state of the system becomes the indirect exciton state. Since the indirect exciton lifetime exceeds the direct exciton one by a few orders of magnitude, the magnetic field can significantly influence optical properties of the system (for example, the luminescence intensity). The realistic range of parameters of a quantum well (well and barrier widths, $\mathrm{Mn}$ and $\mathrm{Mg}$ concentrations, the magnetic field strength), in which the exciton lowest state is the indirect exciton state, is calculated.
\end{abstract}

Keywords: double quantum well, indirect exciton, semimagnetic semiconductor.

Manuscript received 07.02.07; accepted for publication 24.04.07; published online 19.10.07.

\section{Introduction}

A possibility of the binding of an electron and a hole localized in different wells was studied in $[1,2]$. It was shown in [3] that this system has large dipole moment, and, as a result, it should reveal the strong Stark effect. The excitons with spatially separated electrons and holes in two-dimensional semiconductor structures are called indirect excitons, and they recently attracted a great deal of interest [4-9]. The typical method to create indirect excitons is based on the excitation of a double quantum well by light in the presence of an electric field directed along the normal to the plane of the well. The electric field in such systems redistributes the electrons and the holes between different wells and reduces the energy of the indirect exciton level due to the large dipole momentum of these states [7-9]. A specific feature of indirect excitons is the large lifetime caused by the spatial separation of the electron and the hole, and, as the result, a weak overlap of their wave functions. These properties of an exciton make it possible to create a high concentration of excitons and allow investigating the interaction between excitons. Some interesting collective effects in the system of indirect excitons in GaAs-based heterostructures were obtained in recent years [10-13]. But the interpretation of obtained results is often complicated by the presence of the parasitic photocurrent induced by an external electric field. The current leads to the carrier redistribution and changes the potentials and the positions of energy levels in the system. In [14], another method of charge separation in a double quantum well was suggested. The method can be applied to semimagnetic semiconductors, in which a strong exchange interaction between carriers and localized spins of magnetic ions exists, which leads to a strong dependence of the exciton levels on the external magnetic field [15-18]. In this case, the charges can be separated by magnetic field. In [17], two semimagnetic systems based on different materials were calculated. The first system was the (Zn,Be,Mg)Se-based double quantum well system with one semimagnetic well $\mathrm{Zn}_{1-\mathrm{x}} \mathrm{Mn}_{\mathrm{x}}$ Se. It was shown that, in this system at a certain value of the magnetic field, the indirect exciton energy level becomes the lowest one. The second system was a CdTe-based double quantum well with semimagnetic barriers $\mathrm{Cd}_{1-\mathrm{y}} \mathrm{Mn}_{\mathrm{y}} \mathrm{Te}$ and one semimagnetic well $\mathrm{Cd}_{1-\mathrm{x}} \mathrm{Mn}_{\mathrm{x}} \mathrm{Te}$. In this system, two direct exciton levels cross, but the transition from the direct exciton to the indirect one was not found in the magnetic field $B<5$ T.

This article continues paper [17]. We study a more complicated system, in which the CdTe-based system contains $\mathrm{Mn}$ and $\mathrm{Mg}$ ions. The double quantum well 
system with non-magnetic $\mathrm{Cd}_{1-y} \mathrm{Mg}_{\mathrm{y}} \mathrm{Te}$ barriers and one semimagnetic $\mathrm{Cd}_{1-\mathrm{x}} \mathrm{Mn}_{\mathrm{x}} \mathrm{Te}$ well is considered. A presence of $\mathrm{Mg}$ impurities allows tuning the height of the barriers (and, therefore, the energy) and to change a location of single particle levels in the wells. The strong exchange interaction between carriers and the localized spins of magnetic ions of Mn placed in the well leads to a strong dependence of the exciton levels on the external magnetic field. Calculations show that the specially chosen system parameters (well and barrier widths, magnetic ion distribution) allow us to get a structure with the lowest state corresponding to the long-living indirect exciton. This article is devoted to the study of the ranges of inner parameters of the system suitable for the existence of an indirect exciton as the lowest state.

\section{The system under consideration}

The considered structure consists of two CdTe wells, one of which contains the magnetic ions of $\mathrm{Mn}$, and another one contains the non-magnetic impurity, ions of $\mathrm{Mg}$. These non-magnetic and semimagnetic well layers are separated by the non-magnetic CdMgTe barrier layer. The schematic diagram of the heterostructure and its band profiles in an external magnetic field are shown in Fig. 1. The semimagnetic layer forming QW2 provides the possibility to change the well depth for the electron and the hole with an external magnetic field due to the giant Zeeman splitting of the bands [14-18]. The exciton Hamiltonian of the double-well system can be written as

$$
\begin{aligned}
& H=E_{g}-\frac{\hbar^{2}}{2 \mu_{\rho}} \frac{1}{\rho} \frac{\partial}{\partial \rho} \rho \frac{\partial}{\partial \rho}-\frac{\hbar^{2}}{2 m_{e}} \frac{\partial^{2}}{\partial z_{e}^{2}}- \\
& -\frac{\hbar^{2}}{2 m_{h}} \frac{\partial^{2}}{\partial z_{h}^{2}}-\frac{e^{2}}{4 \pi \varepsilon_{0} \varepsilon} \frac{1}{\sqrt{\rho^{2}+\left(z_{e}-z_{h}\right)^{2}}}+ \\
& +V_{e}\left(x_{i}, z_{e}, H, s_{e, z}\right)+V_{h}\left(x_{i}, z_{h}, H, s_{h, z}\right),
\end{aligned}
$$
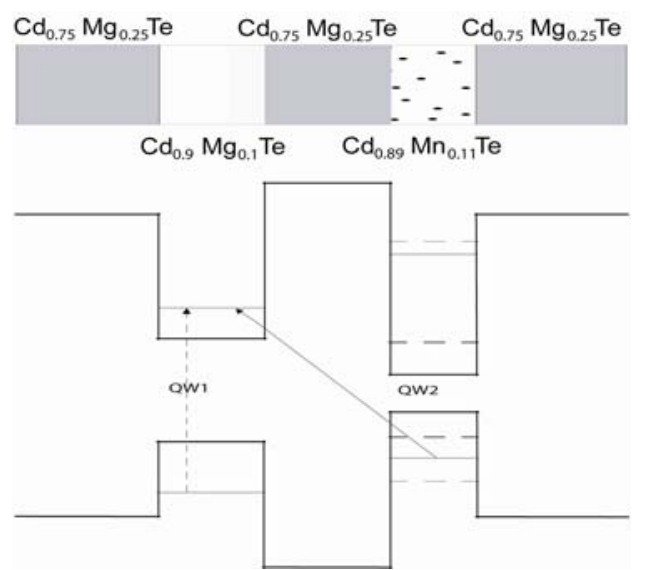

Fig. 1. Schematic diagram of the heterostructures and the band diagram of the double well structure in zero magnetic field (dashed line) and an external magnetic field (solid line). The quantum wells are denoted as QW1 and QW2. Arrows show direct (dashed line) and indirect (solid line) exciton transition. where $E_{g}$ is the bandgap of the narrow-gap semiconductor layer, $z_{e(h)}$ is the electron (hole) position in the direction of the $z$-axis ( $z$ is the direction of the crystal growth), $\rho=\left|\rho_{e}-\rho_{h}\right|, \rho_{e(h)}$ is the electron (hole) position in the plane of the layer, $x_{i}$ is the relative concentration of the impurity ions in the $i$-th layer, $m_{e(h)}$ is the effective mass of the electron (the heavy hole) in the structure growth direction, and $\mu_{\rho}$ is the in-plane reduced exciton mass.

In a general case, the potential $V_{e(h)}\left(x_{i}, z_{e(h)}, H, s_{e(h), z}\right)$ can be divided into two parts. The first part does not depend on the magnetic field, $V_{e(h)}\left(x_{i}\right)=\Omega_{e(h)} \Delta E_{g}$, where $\Delta E_{g}$ is the band offset of two adjacent layers, $\Omega_{e}=\left(1-Q_{V}\right), \Omega_{h}=Q_{V}$, where $Q_{V}$ is the ratio of the valence-band offset to the bandgap difference of the heterojunction. The second part $\quad \Delta V_{e(h)}\left(x_{i}, H, s_{e(h), z}\right)\left[\Theta\left(L_{1}+L_{2}+d-z_{e(h)}\right)-\right.$ $\left.-\Theta\left(L_{1}+d-z_{e(h)}\right)\right]$ describes the exchange interaction and depends on the magnetic field strength $H$ and on the electron (hole) spin projection $S_{e(h), z}[15,17]$. In the diluted magnetic layers, the magnetic field applied along the crystal growth axis changes the band offsets by $\Delta V_{e}=-x N_{0} \alpha\left\langle S_{z}^{\mathrm{Mn}}\right\rangle s_{e, z}$ for the electron and by $\Delta V_{h}=-x \frac{N_{0} \beta}{3}\left\langle S_{z}^{\mathrm{Mn}}\right\rangle S_{h, z}$ for the hole. Here, $N_{0} \alpha$ and $N_{0} \beta$ are the exchange integrals, and $\left\langle S_{z}^{\mathrm{Mn}}\right\rangle$ is the average spin of impurity ions.

We have presented a solution of the Schrödinger equation with Hamiltonian (1) in the form of the superposition of some convenient basic functions

$\Psi=\sum_{i, j=1,2} a_{i j} \Psi_{i j}\left(\rho, z_{e}, z_{h}\right)$.

The basis exciton wave functions were chosen by the way typical of the investigation of quantum wells:

$$
\Psi_{i j}\left(\rho, z_{e}, z_{h}\right)=\varphi_{i}\left(z_{e}\right) \psi_{j}\left(z_{h}\right) \sqrt{\frac{2}{\pi}} \frac{1}{\lambda_{i j}} \exp \left(-\frac{\rho}{\lambda_{i j}}\right),
$$

where $\lambda_{i j}$ are the variational parameters, $\varphi_{i}\left(z_{e}\right)$ and $\psi_{j}\left(z_{h}\right), i, j=1,2$, are the single particle wave functions of an electron (hole) in a single well $i$ ( $j$ ) (i.e. in the absence of another well of the double-well system). The coefficients $a_{i j}$ are the amplitudes of the probability to find an electron in the well $i$ and a hole in the well $j$. In the systems considered here, only the lowest of quantized levels have been taken into account for an electron and a hole in each of the wells. The variation of basis wave functions for an exciton can be chosen in the form 
(3) only in the case where the widths of wells and barriers are much smaller than the exciton radius. The more detailed description of the wave function choice was presented in [17]. The coefficients $a_{i j}$ as well as the eigenvalues of the exciton energy $E_{v}$ were determined by solving the following equation:

$\operatorname{det}|\langle i j|H| k m\rangle-E\langle i j \mid k m\rangle|=0$.

Hereinafter, the exciton energy level $E_{v}$ enumeration starts from the lowest one; $v=1, . ., 4$.

Experimentally the presence of an indirect exciton in the lowest energy level of a double quantum well structures is determined by the increase of the radiation time. The exciton radiation lifetime is inversely proportional to the overlap integral $I_{v}=\left|\int \Psi_{v}(0, z, z) d z\right|^{2}$ that was calculated for each of the exciton states in the double-well structure. Using the value of this integral, we can calculate various optical properties of the system: reflection spectra, absorption and transmission spectra, etc.

\section{Results}

In this work, we have considered the double quantum well structure $\mathrm{Cd}_{0.75} \mathrm{Mg}_{0.25} \mathrm{Te} / \mathrm{Cd}_{0.9} \mathrm{Mg}_{0.1} \mathrm{Te} /$ $\mathrm{Cd}_{0.75} \mathrm{Mg}_{0.25} \mathrm{Te} / \mathrm{Cd}_{0.89} \mathrm{Mn}_{0.11} \mathrm{Te} / \mathrm{Cd}_{0.75} \mathrm{Mg}_{0.25} \mathrm{Te}$. This structure consists of two coupled quantum wells: one is made of a non-magnetic semiconductor, and the other is made of a diluted magnetic semiconductor. The wells are separated by a non-magnetic potential barrier. The bandgap of the CdMgTe layer is taken equal to $E_{g}^{\mathrm{Cd}_{1-\mathrm{x}} \mathrm{Mg}_{\mathrm{x}} \mathrm{Te}}=(1.606(1-x)+3.6 x-0.3 x(1-x)) \mathrm{eV}[19]$, and the bandgap of the CdMnTe layer is equal to $E_{g}^{\mathrm{Cd}_{1-\mathrm{x}} \mathrm{Mn}_{\mathrm{x}} \mathrm{Te}}=(1.606(1-x)+3.198 x) \mathrm{eV}$ [19], where $x$ is the relative impurity concentration. While performing the calculation, the following parameters were used: the effective mass of an electron is $m_{e}=0.096 m_{0}$, the heavy hole mass is $m_{h h}=0.6 m_{0}$; and the dielectric constant $\varepsilon=9.7$ [19]. The exchange integrals are equal to $N_{0} \alpha=0.22 \mathrm{eV}$, $N_{0} \beta=-0.88 \mathrm{eV}, Q_{V}=0.4$ [15]. For the bandgap discontinuity and the average spin projection $\left\langle S_{Z}^{\mathrm{Mn}}\right\rangle$ at $T=1.6 \mathrm{~K}$, we have used the empirical expressions from [20].

Fig. 2 shows the exciton energy level dependence on the applied external magnetic field for the well widths $L_{1}=70 \AA, L_{2}=40 \AA$, and the barrier width $d=50 \AA$ calculated with the use of (4). For the chosen barrier width, the interaction energy of an indirect exciton (of the order of $0.2 \mathrm{meV}$ ) is greater than $k_{\mathrm{B}} T$, which is less than $0.2 \mathrm{meV}$ in the case under

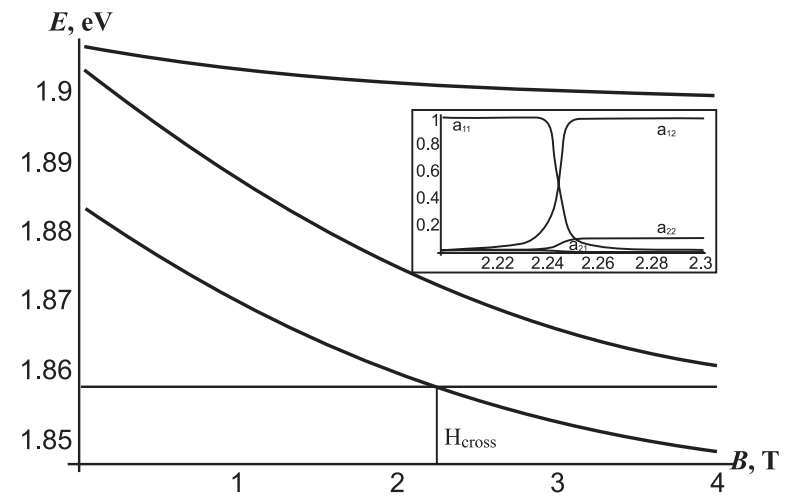

Fig. 2. Magnetic field dependence of the exciton energy spectra.

consideration. The magnetic field value, for which the lowest energy levels $E_{1}$ and $E_{2}$ are maximally close, is denoted in the figure by $H_{\text {cross }}$. The type of the lowest exciton state (direct or indirect) is determined by the values of coefficients $a_{i j}=\left\{a_{11}, a_{12}, a_{21}, a_{22}\right\}$ in the corresponding wave function. The frame in Fig. 2 shows the magnetic field dependence of the coefficients $a_{i j}$ of the lowest energy level. It can be seen that, for $H<H_{\text {cross }}$, the coefficient $a_{11}$ is the largest one. But, for $H>H_{\text {cross }}$, the coefficient $a_{12}$ becomes the largest. In other words, for $H<H_{\text {cross }}$, the lowest state $E_{1}$ corresponds to the exciton with the electron and the hole confined in the same layer (in the non-magnetic well QW1). But, for the $H>H_{\text {cross }}$, it corresponds to the indirect exciton with the electron localized in the well QW1 and the hole placed in the magnetic well QW2. So, an increase in the magnetic field leads to a change of the lowest exciton state spatial characteristics at $H=H_{\text {cross }}$. The gap between these two lowest energy levels at $H=H_{\text {cross }}$ (in the crossing region) is less than $0.05 \mathrm{meV}$, therefore it cannot be observed experimentally. However, the essential increase in the exciton lifetime with change in the external magnetic field can clearly manifest itself in experiment, for example when the luminescence intensity dramatically changes. A similar process was observed experimentally in an electric field in the case of a non-magnetic double quantum well system [6].

Fig. 3 shows the overlap integral dependence on the applied external magnetic field for the exciton energy levels $E_{1}, E_{2}, E_{3}$, and $E_{4}$ denoted as $1,2,3$ and 4 , respectively. The overlap integral value is determined, first of all, by the spatial position of carriers. But, as is clearly seen in the figure, the overlap integral also strongly depends on the applied magnetic field. 


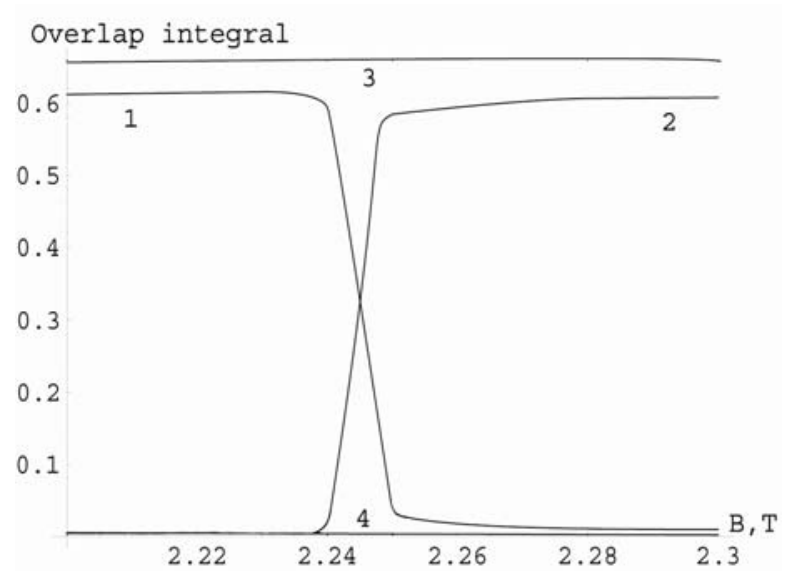

Fig. 3. Overlap integral as a function of the applied magnetic field. Numbers $1, . ., 4$ denote the exciton energy levels (numeration starting from the lowest one).

Overlap integral 3, which corresponds to a direct exciton, exceeds overlap integral 4 of an indirect exciton by a few orders of magnitude. Furthermore, in the presented case, the overlap integrals of two lowest excited energy levels have the step-like dependence on the applied magnetic field. The overlap integral for the lowest exciton state decreases by two orders of magnitude with increase in the magnetic field near $H=H_{\text {cross }}$, whereas the overlap intgral for the second level increases. This behavior of the lowest excited level of the system is caused by a change of the type of the exciton corresponding to this energy level (from direct to indirect one). As mentioned above, the overlap integral is proportional to the oscillator strength of the exciton transition and inversely proportional to the exciton lifetime. According to this, we can conclude that, in the double quantum well system under study, the applied external magnetic field can extremely increase the lifetime of excitons. Experimentally, the presence of the indirect exciton in the lowest energy level of the double quantum well structures leads to an increase in the radiation time.

Quantitatively, the overlap integral depends on the system parameters. It should be mentioned that the indirect exciton state can become the lowest state in such systems, only if certain requirements to the structure are fulfilled. First of all, the width of the barrier placed between the wells must be large enough to provide a maximum localization of the electron and hole wavefunctions. But, in the case of indirect exciton, the Coulomb interaction energy must be greater than $k_{\mathrm{B}} T$. The ranges of the well widths $L_{1}$ and $L_{2}$ for which the indirect exciton in the applied external magnetic field is the lowest state are shown in Fig. 4. The grey region in Fig. 4 corresponds to the situation where, at the magnetic field $B=0$, the lowest energy level belongs to the direct exciton $\left(a_{11}>a_{12}, a_{21}, a_{22}\right)$ and, at the external magnetic

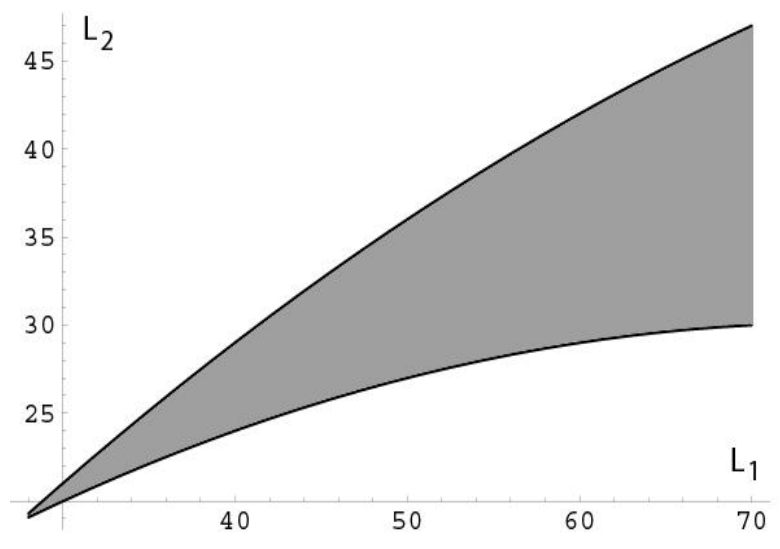

Fig. 4. The range of the well width values, at which we can expect transition of the lowest energy level from the direct exciton to the indirect one. Maximal magnetic field that was taken into account is $5 \mathrm{~T}$.

field $B=5 \mathrm{~T}$, it belongs to the indirect one $\left(a_{12}>a_{11}, a_{21}, a_{22}\right)$.

\section{Conclusion}

The dependence of the exciton energy levels on the applied magnetic field has been studied for the CdTebased double quantum well structure with non-magnetic barriers $\mathrm{Cd}_{1-\mathrm{y}} \mathrm{Mg}_{\mathrm{y}} \mathrm{Te}$. This work shows that, in a certain range of the system parameters, the direct and indirect exciton levels are crossed. Above some critical value of the magnetic field, the indirect exciton becomes the lowest exciton state of the system, which leads to an increase of the exciton lifetime by several orders of magnitude. The present results can be useful for experiments concerning the study of indirect excitons in the double quantum well system and collective exciton effects in semimagnetic semiconductors.

\section{Acknowledgements}

This work was supported by grant INTAS 03-51-5266. The authors wish to thank Dr. O.S. Zinets for the useful discussions.

\section{References}

1. O.S. Zinets, V.I. Sugakov, A.D. Suprun // Fizika, Tekhnika Poluprovodnikov 10, p. 712 (1976) (in Russian).

2. Yu.E. Lozovik, V.I. Yudson // Solid State Communs 19, p. 391 (1976).

3. O.S. Zinets, V.I. Sugakov, A.D. Suprun // Pis'ma Zh. Tekhn. Fiz. 1, p. 61 (1978) (in Russian).

4. T.B. Norris, N. Vodjdani, B. Vinter, C. Weisbuch, G.A. Morrou // Phys. Rev. B 40, p. 1392 (1989).

5. Y.J. Chen, E.S. Koteles, B.S. Elman, C.A. Armiento // Phys. Rev. B 36, p. 4562 (1987). 
6. J.E. Golub, K. Kash, J.P. Harbison, L.T. Florez // Phys. Rev. B 41, p. 8564 (1990).

7. R. Ferreira, G. Bastard // Repts Progr. Phys. 60 (1997).

8. L.V. Butov and A.I. Filin // Phys. Rev. B 58, p. 1980 (1998).

9. Larionov A.V. et al. // JETP Lett. 75, p. 200 (2002).

10. L.V. Butov, A.C. Gossard, and D.S. Chemla // Nature 418, p. 751 (2002).

11. D. Snoke et al. // Nature 418, p. 754 (2002).

12. A.V. Gorbunov, V.B. Timofeyev // Uspekhi Fiz. Nauk 176, p. 651 (2006) (in Russian).

13. A.V. Gorbunov, V.B. Timofeyev // Pis'ma Zh. Tekhn. Fiz. 83 p. 178 (2006) (in Russian).
14. A.V. Komarov, S.M. Ryabchenko, and O.V. Terletskii // Zh. Eksp. Teor. Fiz. 73, p. 608 (1977) (in Russian).

15. J.A. Gaj, R. Planel, and G. Fishman // Solid State Communs 29, p. 435 (1979).

16. P.J. Klar, D. Wolverson, J.J. Davies, W. Heimbrodt, M. Happ // Phys. Rev. B 57, p. 7103 (1998).

17. S.B. Lev, V.I. Sugakov, and G.V. Vertsimakha // J. Phys.: Condens. Matter 16, p. 4033 (2004).

18. D. Keller, D.R. Yakovlev, B. Konig, et al. // Phys. Rev. B 65035313 (2002).

19. W. Ossau et al. // Phys. Low-Dimens. Struct. 11/12 p. 89 (1997).

20. J.A. Gaj et al. // Phys. Rev. B 50 p. 5512 (1994). 